\title{
Determinación del margen terapéutico y estudio de la equivalencia biofarmacéutica de las tabletas multifuentes de digoxina de $0,25 \mathrm{mg}$
}

Ángel Alvarado Yarasca ${ }^{1}$, Lozada Colque Gabriela Laura ${ }^{2}$,Ruth Corazón Llerena Benites ${ }^{2}$,Sadia Ximena Macavilca Francia ${ }^{2}$,Carla Edith Marcos Eguizabal ${ }^{2}$,Nadia Catherine Pisconte Campos ${ }^{2}$, Jonathan Alexander Poma Ghiggo $^{2}$, Hayet Silvia Yunislsmodes Noor ${ }^{2}$

\section{RESUMEN}

Objetivo: Determinar teóricamente el margen terapéutico y experimentalmente, los parámetros de la equivalencia biofarmacéutica de dos lotes de tabletas de multifuentes de digoxina de 0,25 mg.

Material y Métodos: Se estudiarondos lotes, cada una de 200 tabletas de multifuentes de digoxina de 0,25 mg, asignándoles el código de multifuente TDH025lote 105031 y TDF025 lote 10940431. El margen terapéutico teórico se determinó mediante la fórmula farmacocinética VT = Fracción a/ Fracción a predicha $\mathrm{x}$ concentración usual; luego, por diferencia de la CmE y CME se obtuvo el margen terapéutico. El Método analítico utilizado para la cuantificación del principio activo, fue el descrito en la Farmacopea Internacional de la Organización Mundial de la Salud (OMS); la prueba de desgaste por rozamiento y el grado de dureza, se determinó de acuerdo a la USP.

Resultados: el margen terapéutico fue $1,2 \mathrm{ng} / \mathrm{ml}$; los parámetros de la equivalencia biofarmacéutica: porcentajes de principio activo de los dos lotes de medicamentos multifuentes se encontraron dentro del rango de aceptación (90-110\%) propuestos por la USP y la OMS: El desgaste por rozamiento del TDH025 presentó un 0,55\% y el TDF025 tuvo un 0,56\%, ambos valores estuvieron por debajo del 1\% (valor aceptable) y la dureza indicó un soporte de choque mecánico aceptable. Las 06 tabletas se desintegraron completamente, al pasar por un tamiz $\mathrm{N}^{\circ} 10(1700 \mu \mathrm{M})$, en un medio de disolución simulado de $\mathrm{pH}$ gástrico e intestinal en un tiempo menor de 5 minutos.

Conclusión: Se demostró la equivalencia biofarmacéutica de los medicamentos multifuentes de digoxina de 0,25 mg TDH025 lote 105031 y del TDF025 lote 10940431, de acuerdo al criterio de aceptación de la USP y OMS, el principio activo (digoxina) cuantificado en cada lote estuvo dentro del rango de 90-110\%; los parámetros de desgaste por rozamiento y la dureza, indicaron una adecuada estabilidad en su tiempo de vida útil. (Horiz Med 2014; 14(4): 48-52)

Palabras clave: Margen terapéutico, equivalencia biofarmacéutica, medicamento multifuente, digoxina. (Fuente: DeCS BIREME).

\section{Determination of therapeutic range and study of biopharmaceutical equivalence of two multisource Digoxin tablets of $0.25 \mathrm{mg}$}

\section{ABSTRACT}

Objective: To determine the therapeutic safety theoretically and experimentally, the biopharmaceutical equivalence parameters of two batches of multisource Digoxin tablets of $0.25 \mathrm{mg}$.

Material and Methods: Two batches, each of 200 tablets multisource digoxin $0.25 \mathrm{mg}$ were studied, assigning code multisource TDH025 Lot 105031 and TDF025 Lot 10940431. The therapeutic range was determined by theoretical formula $\mathrm{VT}=$ aFraction pharmacokinetics / apredicted fraction by usual concentration $\mathrm{x}$, then by the difference CmE and CME, the therapeutic range was obtained. The analytical method used for quantification of the active ingredient, was described in The International Pharmacopoeia of the World Health Organization (WHO); while the fretting test and the hardness was determined according to the USP.

Results: The therapeutic index is $1.2 \mathrm{ng} / \mathrm{ml}$; and evaluation of the parameters of the biopharmaceutical equivalence percentages of active ingredient of the two lots of multisource drugs are within the acceptance range (90-110\%) given in the USP and WHO. The fretting of TDH025 fretting batch 105031 was $0.55 \%$ and TDF025 was $0.56 \%$, both values are below $1 \%$ which is acceptable value; and hardness indicates an acceptable mechanical shock endurance. The 06 tablets were completely disintegrated, passing through a No. 10 (1700 uM) sieve, in a dissolution medium simulating gastric and intestinal pH in less than 5 min.

Conclusion: Biopharmaceutical equivalence of multisource drugs digoxin $0.25 \mathrm{mg}$ TDH025 TDF025 batch batch 105031 and 10940431 was demonstrated according to the acceptance criteria of the WHO and USP, the active ingredient (digoxin) quantified in each batch were within the range of $90-110 \%$; attrition and hardness parameters indicate adequate stability in their lifetime. . (Horiz Med 2014; 14(4): 48-52)

Key words: Therapeutic range, biopharmaceutical equivalence multisource drug digoxin. (Source: MeSH NLM).

Estudiante de la FMH USMP 


\section{INTRODUCCIÓN}

Para mejorar el acceso a los medicamentos, los genéricos representan una alternativa práctica, por ser económicamente más accesibles y disponibles a sectores más amplios de la población $(1,2)$; pero se cuestiona su eficacia terapéutica (3).

Los medicamentos genéricos, deben tener estudios de bioequivalencia in vitro, a fin de garantizar su actividad terapéutica y ser denominados medicamentos genéricos intercambiables.

La digoxina, es un fármaco con un nivel terapéutico de $0,5-2 \mathrm{ng} / \mathrm{ml}$, por lo que su ventana terapéutica (concentración de un fármaco con elevada probabilidad de éxito terapéutico y baja toxicidad) es de estrecho margen terapéutico $(1,5 \mathrm{ng} / \mathrm{ml})$.

Uno de los parámetros de la equivalencia biofarmacéutica, es la friabilidad, prueba que determina el grado de desgaste de las tabletas durante el transporte y manipulación, la que debe estar en correlación directa con la dureza.

La prueba de contenido de las tabletas, debe estar dentro del rango del porcentaje de aceptación (90$110 \%$ ) descrito en la USP y la Organización Mundial de la Salud (OMS) (4).

La biodisponibilidad de la digoxina es de 50 a $80 \%$ (promedio 70\%), después de la administración de un comprimido por vía oral. Para calcular la dosis, se utiliza su valor promedio (minimiza la posibilidad de sobredosis).

Una vez absorbida, circula unida a la al búmina, entre 20 a 30\% (promedio 25\%) Su fracción libre, sigue un modelo farmacocinético bicompartimental.

El tiempo de vida media $a$, es de 35 minutos, el tiempo de vida media $B$ es de 1,5-2 días (36-48 horas), en pacientes con función renal normal. Ello permite administrar una dosis al día.

En personas sanas, su depuración metabólica es de 0,57 a $0,86 \mathrm{~mL}$. $\mathrm{min}-1 / \mathrm{kg}$.

Se excreta un $30 \%$ en forma de fármaco inalterado por vía biliar; y por su coeficiente de partición polar, se excreta en forma de fármaco inalterado en un $50-80 \%$, dentro de las 6 a 12 horas después de una dosis intravenosa. En personas sanas, la eliminación diaria renal, representa el 33\% de sus depósitos corporales, y es proporcional a la tasa de filtración glomerular, por lo que la dosis de mantenimiento deberá calcularse en función de la depuración de creatinina $(5,6)$.

Esta investigación, evalúa la bioequivalencia in vitro de la digoxina genérica que se expende en el mercado peruano.

\section{MATERIAL Y MÉTODOS}

Estudio de tipo experimental, descriptivo, cuantitativo, prospectivo.

Las muestras estudiadas fueron de origen nacional, las cuales consistieron en dos lotes, cada una de 200 tabletas de multifuentes de digoxina de 0,25 mg, asignándoles el código de multifuente TDH025 lote 105031 y TDF025 lote 10940431 .

El margen terapéutico teórico, se determinó mediante la fórmula farmacocinética VT = Fracción a/ Fracción a predicha $x$ concentración usual; luego por diferencia de la CmE y CME se obtuvo el margen terapéutico.

El Método analítico utilizado para la cuantificación del principio activo fue el descrito en la Farmacopea Internacional de la Organización Mundial de la Salud (OMS); mientras que la prueba de desgaste por rozamiento y el grado de dureza se determinó de acuerdo a la USP.

El tiempo de desintegración se determinó, en dos medios de $\mathrm{pH}$, que simulan el medio gástrico ( $\mathrm{pH}$ 1,2) e intestinal $(\mathrm{pH} 7,0)$, adaptado del reporte técnico 937 de la OMS.

\section{RESULTADOS}

Utilizando la fórmula de ventana terapéutica se reportó una concentración plasmática mínima de $0,8 \mathrm{ng} / \mathrm{ml}$ y la máxima efectiva de $2 \mathrm{ng} / \mathrm{ml}$. 
Ángel Alvarado Yarasca, Lozada Colque Gabriela Laura, Ruth Corazón Llerena Benites, Sadia Ximena

Macavilca Francia, Carla Edith Marcos Eguizabal, Nadia Catherine Pisconte Campos, Jonathan Alexander Poma Ghiggo, Hayet Silvia Yunislsmodes Noor

La ventana terapéutica se dedujo a partir de los niveles terapéuticos, hallándose un valor de $1,2 \mathrm{ng} / \mathrm{ml}$. Tabla 1.

Tabla 1.- Determinación de la ventana terapéutico de la tableta de digoxina de $0,25 \mathrm{mg}$

\begin{tabular}{ccc}
\hline DCl & $\begin{array}{c}\text { Niveles } \\
\text { terapéuticos } \\
(\mathbf{n g} / \mathbf{m l})\end{array}$ & $\begin{array}{c}\text { Ventana } \\
\text { terapéutica } \\
(\mathbf{n g} / \mathbf{m l})\end{array}$ \\
\hline Digoxina & $0,8-2$ & 1,2 \\
\hline
\end{tabular}

Con la prueba de contenido de las tabletas se comprobó que ambas multifuentes se encontraban dentro del rango de aceptación propuesto en la USP y OMS de $90-110 \%$. Tabla 2.

Tabla 2.- Prueba de contenido de las tabletas multifuentes de digoxina de $0,25 \mathrm{mg}$

\section{Multifuentes en estudio} $(n=3)$

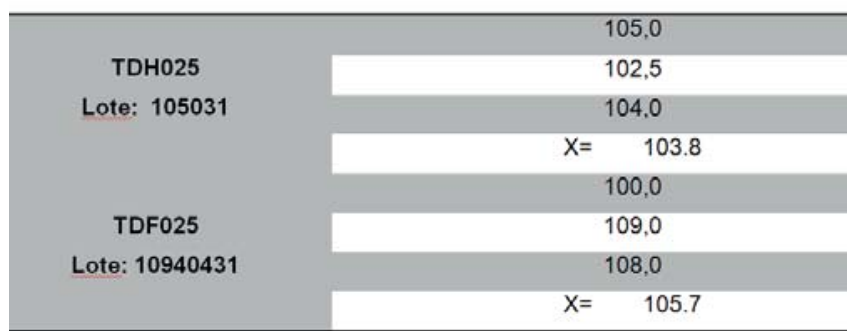

El porcentaje de desgaste por rozamiento, tras pesar 20 tabletas de cada lote, se encontró por debajo del 1\%. Tabla 3.

Los valores resultantes de la prueba de dureza se reportan en la Tabla 4.

La velocidad de desintegración se determinó en función del tiempo y a dos pH diferentes, observándose que se desintegraron antes de los 30 minutos. Tabla 5.

\section{DISCUSIÓN}

Esde vital importancia, comprobar la bioequivalencia farmacéutica entre medicamentos que contienen el mismo principio activo, asegurando así la eficacia terapéutica sin importar el uso intercambiable de los mismos, aspecto muy importante a considerar durante el manejo de fármacos en la actividad médica (4).
Tabla 3. - Determinación del porcentaje de desgaste por rozamiento de las tabletas multifuentes de digoxina de $0,25 \mathrm{mg}$

\begin{tabular}{|c|c|c|c|}
\hline $\begin{array}{l}\text { Multifuente } \\
\text { en estudio }\end{array}$ & $\begin{array}{c}\text { Peso } \\
\text { inicial } \\
\text { de } 20 \\
\text { tabletas } \\
\text { (g) }\end{array}$ & $\begin{array}{c}\text { Peso } \\
\text { final de } \\
20 \\
\text { tabletas } \\
\text { (g) }\end{array}$ & $\begin{array}{c}\text { Porcentaje } \\
\text { de } \\
\text { desgaste } \\
(<1 \%)\end{array}$ \\
\hline \multirow{4}{*}{$\begin{array}{l}\text { TDH025 } \\
\text { Lote: } \\
105031\end{array}$} & 1,503 & 1,496 & 0,466 \\
\hline & 1,503 & 1,494 & 0,599 \\
\hline & 1,502 & 1,493 & 0,599 \\
\hline & & $X=0,55$ & \\
\hline \multirow{4}{*}{$\begin{array}{l}\text { TDF025 } \\
\text { Lote: } \\
10940431\end{array}$} & 2,006 & 1,997 & 0,448 \\
\hline & 2,010 & 1,994 & 0,796 \\
\hline & 2,008 & 1,999 & 0,448 \\
\hline & \multicolumn{3}{|c|}{$X=0,564$} \\
\hline
\end{tabular}

Tabla 4.- Determinación del grado de dureza de las tabletas multifuentes de digoxina de $0,25 \mathrm{mg}$

\begin{tabular}{|c|c|c|c|}
\hline \multicolumn{2}{|c|}{$\begin{array}{l}\text { Multifuente } \\
\text { TDH025 } \\
\text { Lote: } 105031\end{array}$} & \multicolumn{2}{|c|}{$\begin{array}{l}\text { Multifuente } \\
\text { TDH025 } \\
\text { Lote: } 10940431\end{array}$} \\
\hline Tabletas & Dureza & Tabletas & Dureza \\
\hline M-01 & 1,408 & M-01 & 0,845 \\
\hline M-02 & 1,267 & M-02 & 0,845 \\
\hline M-03 & 1,759 & M-03 & 0,845 \\
\hline M-04 & 1,408 & M-04 & 0,845 \\
\hline M-05 & 1,408 & M-05 & 0,845 \\
\hline M-06 & 1,408 & M-06 & 0,845 \\
\hline M-08 & 1,408 & M-08 & 0,845 \\
\hline M-09 & 1,267 & M-09 & 0,845 \\
\hline M-10 & 1,267 & $M-10$ & 0,845 \\
\hline$M-11$ & 1,267 & $\mathrm{M}-11$ & 0,845 \\
\hline M-12 & 1,267 & M-12 & 0,845 \\
\hline M-13 & 1,759 & M-13 & 0,845 \\
\hline M-14 & 1,759 & $\mathrm{M}-14$ & 0,845 \\
\hline$M-15$ & 1,759 & $M-15$ & 0,845 \\
\hline$M-16$ & 1,759 & $M-16$ & 0,845 \\
\hline $\mathrm{M}-17$ & 1,408 & $\mathrm{M}-17$ & 0,845 \\
\hline M-18 & 1,408 & M-18 & 0,845 \\
\hline$M-19$ & 1,408 & M-19 & 0,845 \\
\hline M-20 & 1,408 & $M-20$ & 0,845 \\
\hline Dureza X: & 1,249 & Dureza $X$ : & 1,249 \\
\hline
\end{tabular}


Tabla 5. Determinación del tiempo de desintegración de las tabletas multifuentes de digoxina de $0,25 \mathrm{mg}$

\begin{tabular}{|c|c|c|}
\hline \multicolumn{3}{|c|}{ Tiempo de desintegración a diferentes pH (min) } \\
\hline Multifuentes & $\mathrm{pH} \mathrm{1,2}$ & pH 7,00 \\
\hline \multirow{6}{*}{$\begin{array}{c}\text { TDH025 } \\
\text { Lote: } 105031\end{array}$} & $4: 15$ & $1: 11$ \\
\hline & $3: 18$ & $1: 08$ \\
\hline & $4: 08$ & $1: 04$ \\
\hline & $3: 15$ & 1:01 \\
\hline & $4: 35$ & $1: 11$ \\
\hline & $3: 45$ & $1: 08$ \\
\hline \multirow{8}{*}{$\begin{array}{c}\text { TDH025 } \\
\text { Lote: } 10940431\end{array}$} & $X=4,53$ & $X=1: 08$ \\
\hline & $5: 17$ & $1: 17$ \\
\hline & $4: 19$ & $1: 13$ \\
\hline & $4: 15$ & $1: 09$ \\
\hline & $4: 27$ & $1: 07$ \\
\hline & $5: 30$ & $1: 03$ \\
\hline & $4: 12$ & $1: 00$ \\
\hline & $X=4,53$ & $X=1: 08$ \\
\hline
\end{tabular}

En nuestros resultados, encontramos como margen terapéutico: 1,2 ng/ $\mathrm{ml}$, lo que concuerda con el típico rango terapéutico de la digoxina considerado entre 0,5-2 $\mathrm{ng} / \mathrm{ml}$. Sin embargo, podemos citar la investigación de Medeiros, et al, acerca de la monitorización terapéutica de la digoxemia en pacientes con IC donde remiten que los niveles terapéuticos de la digoxina presentan alto grado de variabilidad y muchas veces se sobreponen a los de la toxicidad. Por lo que, una digoxinemia inferior a $0,5 \mathrm{ng} / \mathrm{mL}$ puede presentarse con una respuesta terapéutica adecuada y una digoxinemia superior a $2,0 \mathrm{ng} / \mathrm{mL}$ no condiciona necesariamente la presencia de signos o síntomas de intoxicación digitálica (7). Por ello, ante la divergencia de opiniones, sería recomendable monitorizar las concentraciones plasmáticas de la digoxina a fin de evitar la toxicidad de los fármacos que pueden llevar a una arritmia cardíaca.

Si bien el rango general de $0.5-2 \mathrm{ng} / \mathrm{mL}$ es útil para la mayoría de pacientes, en ciertas condiciones, la ventana terapéutica se podría ajustar fuera de él, como lo menciona Jelliffe RW en su estudio sobre Digitálicos que se asocian con la conversión al ritmo sinusal en pacientes con fibrilación auricular (FA), donde dedujo que los individuos con FA y adecuada conducción aurículoventricular, requieren valores más elevados de digoxina sérica, por lo cual podría postularse un objetivo terapéutico mayor: 1.5 a 2.4 $\mathrm{ng} / \mathrm{ml}(8)$.

El porcentaje del contenido de las tabletas multifuentes de digoxina estudiadas, se encuentran dentro del rango de aceptación: $90-110 \%$, propuesto por la Farmacopea de los Estados Unidos de América (USP), lo que nos demuestra una equivalencia farmacéutica (9). En los reportes revisados, se indica que en fármacos altamente solubles, la bioequivalencia in vitro es criterio suficiente para comparar el medicamento multifuente con el innovador (10). Cabe resaltar además, que los estudios in vitro son considerablemente menos costosos y nos permiten predecir los resultados in vivo (11).

Dado que el Porcentaje de pérdida de peso obtenido en 2 Lotes analizados de digoxina de 0.25 mg fue inferior a $1 \%$, se puede concluir que cumplen con el criterio de Friabilidad. Estos resultados nos indican que las tabletas, poseen una buena resistencia al desgaste sufrido durante la manipulación, envasado y transporte (12).

Respecto a los resultados de la prueba de dureza, se encontró que las tabletas multifuentes TDF025 presentaban un menor valor, lo cual indica la presencia de poco aglutinante o una débil compresión que puede acelerar la disolución del principio activo; a comparación de la tableta multifuentes TDH025 que tuvo un grado de dureza superior, lo cual nos indica o una mayor presencia de aglutinante o una compresión mayor de la máquina tableteadora, lo cual pudiera disminuir el perfil de disolución del principio activo, así como la biodisponibilidad del mismo (13).

Adicionalmente, a la equivalencia biofarmacéutica, hemos investigado el tiempo de desintegración a un $\mathrm{pH}$ simulado al medio gástrico $(\mathrm{pH} 1,2)$ y al intestinal ( $\mathrm{pH} 7)$, encontrando que ambos lotes en estudio, se desintegraron en menos de $30 \mathrm{~min}$, lo que indica una elevada concentración de los desintegrantes durante el proceso de manufactura, lo que puede ser un problema terapéutico, ya que según un estudio realizado en Reino Unido por Shaw, existe una estrecha correlación entre la velocidad de disolución de las tabletas y los niveles de digoxina en plasma. 
La medición de la velocidad de disolución in-vitro parece ser un método válido para garantizar que las diferentes tabletas de digoxina son de igual eficacia. Sin embargo, en algunos pacientes, la absorción del fármaco es notablemente sensible a cambios en la velocidad de disolución y nuevos estándares de farmacopea no deben ser definidos hasta que formulaciones de disolución rápida se hayan estudiado. De lo contrario, se podría sobrepasar la concentración máxima efectiva (CME) o mínima tóxica y desencadenar arritmias cardíacas (14). Por ello, proponemos que se hagan estudios de la cinética de disolución para corroborar la velocidad de desintegración de nuestro estudio.

En conclusión, se demostró la equivalencia biofarmacéutica de los medicamentos multifuentes de digoxina de 0,25 mg TDH025 lote $105031 \mathrm{y}$ del TDF025 lote 10940431, de acuerdo al criterio de aceptación de las Farmacopeas Oficiales USP y OMS.

El principio activo (digoxina) estuvo dentro del rango aceptado, al igual que los parámetros de desgaste por rozamiento y dureza, lo cual indica una adecuada estabilidad en su tiempo de vida útil.

\section{Fuentes de financiamiento}

Este estudio ha sido autofinanciado por el autor.

\section{Conflictos de interés}

El autor declara no tener ningún conflicto de interés.

\section{Correspondencia:}

Ruth Corazón Llerena Benites

Dirección: Mz W, Lt 17,Jr. Alicante. La Molina. Lima Teléfono: (51) 95088769

Correo electrónico: rcllb@hotmail.com

Recibido: 24 de Julio de 2014 Aprobado: 06 de Octubre de 2014

\section{REFERENCIAS BIBLIOGRÁFICAS}

1. AIS. Acción internacional para la salud. Genéricos y bioequivalencia. Balance y perspectivas América Latina. Lima.2004.

2. Programa de Medicamentos Esenciales y Tecnología.Consulta de Expertos en Bioequivalencia de Productos Farmacéuticos. 13-15 de enero de 1999. Caracas: Organización Panamericana de la Salud. Informe final; Junio de 1999.

3. AIS. Acción Internacional para la Salud. Genéricos e Intercambiabilidad. Boletín AIS-LAC. Lima. Marzo 2001.

4. Organización Panamericana de la Salud. Criterios científicos para los ensayos de bioequivalencia (in vivo e in vitro), las bioexenciones y las estrategias para su implementación: documento borrador. En: IV Conferencia Panamericana para la Armonización de la Reglamentación Farmacéutica. RepúblicaDominicana 2-4 de Marzo, 2005.

5. Velasco A, San Román L, Serrano J, Martínez R, Cadavid M. Farmacología Fundamental. Madrid: McGraw-Hill Interamericana; 2002.

6. Goodman A, Hardman J, Limbird L, Molinoff P, Ruddon R. Las Bases Farmacológicas de la Terapéutica. 11 ed. México: McGrawHill Interamericana; 2006.

7. Medeiros Barros I, Marra da Madeira Freitas E, Rassi S, Carneiro J, Rego C, Deus Honório T, et al. Monitorización terapéutica de la digoxinemia en pacientes con insuficiencia cardiaca: comparación entre dos métodos analíticos. FarmHosp. [Internet]. 2014 Feb [citado 10 Nov 2014]; 38(1): 38-43. Disponible en: http://scielo. isciii.es/scielo. php?pid=S1130-63432014000100006\&script $=$ sci_ arttext

8. Jelliffe RW. The role of digitalis pharmacokinetics in converting atrial fibrillation and flutter to regular sinus rhythm. ClinPharmacokinet. 2014;53(5):397-407.

9. Farmacopea de los Estados Unidos de América USP 36 -NF 31, Formulario Nacional, edición 35, volumen 1, 2 y 3, TheUnitedStatesPharmacopeialConvention, Rockville, MD 2013

10. Huayanay-Falconi, L. Bioequivalenciaen medicamentos. RevMedHered[internet]. 2012[citado 2014-11-09], 23(4): 221-222

11. Chen L, Shah VP, Crommelin DJ, Shargel L, Bashaw D, Bhatti M, et al. Harmonization of Regulatory Approaches for Evaluating Therapeutic Equivalence and Interchangeability of Multisource Drug Products: Workshop Summary Report. The AAPS Journal 2011; 13(4): 556-564.

12. Diana M. Albanes A., Brenda L. Valiente Z. Correlación de la calidad fisicoquímica-precio de dos analgésicos comercializados en el municipio de Sonsonate. San Salvador. 2012.

13. Martínez M, Camacho I, Gracia Y, Gracia S. Evaluación invitro de doce marcas de comprimidos de ciprofloxacina que se comercializan en el mercado mexicano. Rev. Mexicana de Ciencias Farmacéuticas. 2010;41(4):43-49.

14. Shaw T, Raymond K., Howard M, Hamer J. Therapeutic Nonequivalence of Digoxin Tablets in United Kingdom: Correlation with Tablet Dissolution Rate. Br Med J 1973; 4:763. 